\title{
CD97 Is Decreased in Preeclamptic Placentas and Promotes Human Trophoblast Invasion Through PI3K/Akt/mTOR Signaling Pathway

\author{
Huaxiang Shen, MD ${ }^{1,2}$, Minfei Jin, MD', Shengyi Gu, BA', \\ Yuelin Wu, MA', Mengnan Yang, MA', and Xiaolin Hua, MD, PhD'
}

\begin{abstract}
Preeclampsia $(\mathrm{PE})$ is a pregnancy disorder leading to the morbidity and mortality. Despite the development of the understanding of etiology, the only effective treatment of PE is the delivery of the placenta. An improved mastery on the regulation of trophoblast invasion could be meaningful to alleviate the disease burden of PE. Relative expression of CD97 in PE and normal placental tissues was evaluated by quantitative real-time polymerase chain reaction, immunohistology, and Western blot. The CD97 siRNA and expression vector was transfected to cultured human trophoblast HTR-8/SVneo, and the cell invasion as well as the protein expression in PI3K/Akt/mTOR signaling pathway were evaluated. Expression of CD97 is significantly downregulated in PE placental tissues compared to normal controls. The Si-CD97 inhibits HTR-8/SVneo trophoblast cells invasion, as well as the activation of PI3K/Akt/mTOR signaling pathway. In accordance, overexpression of CD97 promotes trophoblast cell invasion. In addition, CD97 regulates FOXC2 expression and showed similar effects on PI3K/Akt/mTOR signaling pathway as specific FOXC2 inhibitor. In short, this study demonstrated the downregulation of CD97 expression in preeclamptic placentas. Further mechanism investigation revealed that CD97 promoted trophoblast invasion by targeting FOXC2 via PI3K/Akt/mTOR signaling pathway, laying the foundation for the development of PE intervention strategy by targeting CD97 in placentation and pathogenesis of PE.
\end{abstract}

\section{Keywords}

preeclampsia, CD97, PI3K/Akt/mTOR, trophoblast invasion, FOXC2

\section{Introduction}

Preeclampsia (PE) is a pregnancy disorder that is mainly characterized by hypertension and significant proteinuria starting at 20 weeks of gestation. This complication affects both mother and fetus, with $3 \%$ to $8 \%$ of incidence among pregnant women worldwide ${ }^{1}$ In brief, PE pathogenesis involves 2 stages, while the first stage is characterized by a defective placentation and the second stage is characterized by maternal endothelial damage and systemic inflammation ${ }^{2}$ Preeclampsia is a multifactorial syndrome impacted by angiogenesis, immunological changes, genetic factors, oxidative stress, and abnormal placentation and placental implantation ${ }^{3-7}$ For instance, several in vitro and in vivo studies have highlighted the importance of hypoxia-inducible factor, which affects placental development via regulating the transforming growth factor, vascular endothelial growth factor, and erythropoietin in the maternal circulation $^{3}$ In addition, abnormal immune responses have been found in the placental microenvironment of PE patients, and the various components of the immune system including interferon $\gamma$ and transforming growth factor $\beta$ cytokines have been reported to participate in the early pregnancy events by inhibiting cytotrophoblast invasion ${ }^{4}$ Moreover, extensive attention has been given to possible maternal $\mathrm{T}$-cell responses to fetal histocompatibility since placental trophoblast cells do not express the strong histocompatibility antigens ${ }^{5}$ Oxidative stress could also play a key role in the pathology of PE both in the placenta and in the maternal endothelium ${ }^{6}$

In spite of the development of various hypotheses toward the etiology of PE, the precise pathogenesis is not fully understood. Currently, the only effective treatment occurs when the placenta is delivered, ${ }^{8}$ and thus, it is not difficult to acknowledge that the early success of a pregnancy relies on appropriate

\footnotetext{
' Department of Obstetrics and Gynecology, Xinhua Hospital, Shanghai Jiaotong University School of Medicine, Shanghai, People's Republic of China

${ }^{2}$ Department of Obstetrics, Jiaxing Maternity and Child Health Hospital, Jiaxing, Zhejiang, People's Republic of China
}

\section{Corresponding Author:}

Xiaolin Hua, Department of Obstetrics and Gynecology, Xinhua Hospital, Shanghai Jiaotong University School of Medicine, Shanghai 200092, People's Republic of China.

Email: xiaolinha@sina.com 
human placental development, especially implantation and placentation ${ }^{9}$ Human placental development is critical for embryonic development and successful pregnancy. Following implantation, the invasion of trophoblasts has been indicated to play roles in the pathogenesis of $\mathrm{PE}^{10}$ The CD97, which belongs to the epidermal growth factor-7-transmembrane subfamily, has been found to promote proliferation and invasion of gastric cancer cells through exosome-mediated mitogen-activated protein kinases signaling pathway $^{11}$ Moreover, CD97 localizes with invadopodia, the actin-rich structures which are proposed to participate in epithelial-mesenchymal transition and glioblastoma multiform tumor invasion ${ }^{12}$ CD97 was originally found to be expressed by hematopoietic cells, and previous studies demonstrated CD97 was associated with increased invasion of gastric cancer, thyroid carcinoma, and colorectal carcinoma ${ }^{13,14}$ Specifically, CD97 expression was found in $93 \%$ of the colorectal adenocarcinomas in a study that included 83 clinical samples by immunohistology analysis, and the strongest staining for CD97 was observed in scattered tumor cells at the invasion front ${ }^{13}$ Further, using knockdown and orthotopic gastric cancer mouse model, CD97 small isoform was found to support the local growth of gastric carcinomas, promoting the tumor development and metastasis ${ }^{14}$

With respect to the proliferative and invasive characterizations, trophoblasts and tumor cells share similarities. Herein, we aimed to investigate the expression alteration of CD97 in the placental tissue of PE patients, as well as the potential regulatory role of $\mathrm{CD} 97$ in the pathogenesis of $\mathrm{PE}$.

\section{Materials and Methods}

\section{Clinical Specimen Collection}

Human placental tissue samples were obtained from patients with PE diagnosed at Jiaxing Maternity and Child Health Hospital according to American College of Obstetricians and Gynecologists. The clinical characters of the participants can be found in Table 1. Placental tissues from healthy pregnancy volunteers were also collected from the same hospital. The protocol was approved by the institutional ethics committee, and informed consent was obtained from each patient. Three pieces of tissues at approximately $1 \times 1 \times 1 \mathrm{~cm}^{3}$ were resected midway between the chorionic and basal plates of the newly delivered placentas during cesarean section. The collected tissues were rinsed with ice-cold phosphate-buffered saline (PBS), 2 of which were snap-frozen in liquid nitrogen and stored at $-80^{\circ} \mathrm{C}$ for subsequent use and the other one was fixed in $4 \%$ formaldehyde.

\section{Cell Line and Culture}

The human trophoblast cell line HTR-8/SVneo was purchased from Shanghai Shybio Co, Ltd (Shanghai, China). The cells were cultured in RPMI 1640 (Gibco, Maryland) supplemented with $10 \%$ (vol/vol) fetal bovine serum (FBS; Gibco) at $37^{\circ} \mathrm{C}$ in an atmosphere of $5 \% \mathrm{CO}_{2}$.

\section{Quantitative Real-Time PCR}

Total RNA was extracted from excised placenta tissues and cultured trophoblast cells using TRIzol (Invitrogen, Carlsbad, California), according to the manufacturer's protocol. Complimentary DNA was synthesized using a reverse transcription kit (TaKaRa, Dalian, China) and then stored at $-20^{\circ} \mathrm{C}$ until use. Quantitative real-time PCR (qRT-PCR) was performed on an ABI 7500 Real-Time PCR System (Applied Biosystems, Waltham, MA) using the PCR primers as following: CD97, 5'-ACTCTGCCGGGAGCTGAAAC-3' (forward); 5'-TGG ATGGTGACCTCGGCTGA-3' (reverse); and GAPDH, 5'-AGA AGG CTG G GG CTC ATT TG-3' (forward), 5'-A GG GGC CAT CCA CAG TCT TC-3' (reverse). Relative quantification of PCR products was based on the differences in $\mathrm{Ct}$ values between CD97 gene and the housekeeping gene GAPDH using the $\triangle \triangle \mathrm{Ct}$ method. All experiments were performed in triplicate.

\section{Western Blot Assay}

For the protein expression analysis of CD97, equal amounts of protein $(20 \mu \mathrm{g})$ from the lysates of placenta tissue and HTR-8/ SVneo cells were separated by $12 \%$ sodium dodecyl sulfate polyacrylamide gel electrophoresis and transferred to polyvinylidene difluoride membranes (Bio-Rad, Hercules, CA). The membranes were blocked in $5 \%$ nonfat dry milk in Tris-Buffered Saline and Tween 20 (TBST, 0.1\% Tween 20) for 2 hours at room temperature, followed by the incubation with primary monoclonal antibodies against CD97 (1:5000) and $\beta$-actin (1:2000; Santa-Cruz Biotech, Dallas, TX) overnight at $4^{\circ} \mathrm{C}$. Membranes were washed 3 times with TBST and incubated with secondary HRP-conjugated rabbit anti-mouse immunoglobulin G (1:20 000; Sigma-Aldrich, St. Louis, MO) at room temperature for 1 hour. All membranes were detected using enhanced chemiluminescence (Thermo Fisher Scientific, Waltham, MA) and the density of each band was analyzed by ImageJ (National Institutes of Health, Bethesda, Maryland). In addition, for signaling pathway analysis, antibodies against phosphatidylinositol-3-kinase (PI3K), p-Akt, and the mammalian target of rapamycin (pmTOR) were used for Western blot as described above.

\section{Immunohistochemical Detection of CD97}

Formalin-fixed placenta tissues were sectioned (5- $\mu \mathrm{m}$ thickness), mounted, and air-dried. After washing with PBS, the sections were incubated with antibodies against mouse CD97, followed by the streptavidin ebiotin-peroxidase complex method using a SP Rabbit horseradish peroxidase Kit (CWbio, Beijing, China). Images captured at $200 \times$ magnification were acquired using an Olympus microscope (Olympus, Tokyo, Japan). The mean intensity of each staining was processed with automated analysis using Image-Pro Plus software version 6.0 (Media Cybernetics, Maryland). The average mean intensity (unit) of 5 random fields were calculated and expressed as the mean (standard deviation [SD]). 


\section{Matrigel Invasion Assay}

Matrigel invasion assay was performed to investigate the effect of CD97 on HTR-8/SVneo cell invasion. Briefly, HTR-8/ SVneo cells were suspended at 1:3 dilution with medium and seeded at $50 \mu \mathrm{L} /$ well in the Matrigel-coated upper chambers of 24-well Transwell invasion plates (BD Biosciences, Franklin Lakes, NJ). RPMI 1640 containing $10 \%$ FBS was added to the lower chamber. The cells were incubated for 30 hours at $37^{\circ} \mathrm{C}$, and noninvaded cells on the upper surface of the chamber were wiped. Invaded cells at the lower face of the chamber were fixed in $4 \%$ paraformaldehyde, stained with $0.1 \%$ crystal violet, and counted in 10 random fields $(200 \times)$. The data are presented from 3 independent experiments.

\section{Knockdown and Overexpression of CD97 in HTR-8/SVneo Cells}

For knockdown study, CD97 siRNAs (si-CD97-1 and si-CD972) and nontargeting siRNA control (si-scramble) were purchased from Shanghai Genepharma Inc (Shanghai, China) and delivered to HTR-8/SVneo cells.

For overexpression study, CD97 expression and control vector plasmids were purchased from ViGene Biosciences, Inc (Shandong, China) and transfected into HTR-8/SVneo cells using Lipofectamine 2000 (Thermo Fisher Scientific). Following an additional 24-hour culture after transfection, the cells were harvested and used in subsequent assays.

\section{Inhibition and Knockdown of PI3K/Akt/mTOR Components and FOXC2}

The PI3K/mTOR inhibitor nVP-BeZ235 (SelleckChem, Shanghai, China) and FOXC2 siRNA (Si-FOXC2, purchased from Shanghai Genepharma Inc) were used to treat HTR-8/ SVneo cells, respectively. The expression of PI3K/Akt/mTOR components and FOXC2 were confirmed by Western blot, and the cell invasion of HTR-8/SVneo cells was assessed by Matrigel invasion assay as described earlier.

\section{Statistical Analysis}

The data were analyzed using SPSS software version 20.0 (SPSS Inc, Chicago, Illinois). All data were presented as mean (SD) from at least 3 independent experiments. Student $t$ test was used for comparison between groups. $P$ values $<.05$ were considered statistically significant.

\section{Results}

\section{Preeclampsia Placentas Demonstrated Lower CD 97 Expression}

Quantitative RT-PCR results showed that the mRNA expression level of CD97 in PE placentas was significantly downregulated in comparison with placentas of healthy control group (Figure 1A). In addition, CD97 localized in the midway between the chorionic and basal plates as shown in the immunohistochemical assay. The CD97 immunostaining in placentas from the PE was also downregulated (Figure 1B), while the mean immunostaining intensity in PE group was as low as $25 \%$ of the staining level of CD97 in healthy normal groups (Figure 1C). Likewise, Western blot result showed that relative CD97 protein expression normalized to $\beta$-actin in PE group was significantly lower than the one in healthy control group (Figure $1 \mathrm{D}$ and $\mathrm{E})$.

\section{Si-CD97 Inhibits HTR-8/SVneo Trophoblast Cell Invasion}

To identify the effects of CD97 in the pathological progression of PE, we investigated the role of CD97 on trophoblast cells invasion using HTR-8/SVneo cell line. Firstly, 2 different CD97 siRNAs (Si-CD97) were transfected into the culture cells, and qRT-PCR results showed that both si-CD97-1 and si-CD97-2 decreased relative CD97 mRNA levels, while the value in si-CD97-2-treated cells was significantly lower than nontargeting siRNA control $(P<.05$; Figure $2 \mathrm{~A})$. Further, Transwell invasion assay showed that the invasion of trophoblast cells was inhibited by CD97 silencing (Figure 2C), and statistical analysis showed that the number of invading cells in si-CD97-2 group was significantly reduced to approximately $30 \%$ of the negative control group (Figure 2B).

\section{CD97 Overexpression Promotes Trophoblast Cells Invasion}

To validate the modulatory function of CD97 in trophoblast invasion, CD97 was overexpressed in HTR-8/SVneo cells, followed by the Transwell invasion assay. As presented in Figure 3A, CD97 mRNA level was significantly higher than the one in the control group, indicating the stable overexpression of CD97. Consistent with the knockdown assay, the invasive capabilities of trophoblast cells were promoted in the cells with CD97 overexpression (Figure 3C). The number of invading cells in the CD97 overexpression group was significantly elevated than empty vector control group (Figure 3B), suggesting the enhancement of CD97 on human trophoblast invasion.

\section{CD97 Regulates the PI3K/Akt/mTOR Signaling Pathway}

Several previous studies have shown that PI3K/Akt signaling pathway was relevant to trophoblast invasion. ${ }^{15-17}$ Therefore, we further assessed the regulation of CD97 on this specific pathway to reveal the potential mechanism of action of $\mathrm{CD} 97$ on trophoblast invasion. As seen in Figure 4A and B, we found HTR-8/SVneo cells with low level of CD97 by si-CD97 transfection showed significantly downregulated protein levels of PI3K, p-Akt, and p-mTOR $(P<.05)$.

\section{CD97 Regulates FOXC2 Expression}

It has been illustrated that FOXC2 could promote the invasion ability of normal trophoblast cells, ${ }^{18}$ and we further explore the 
A

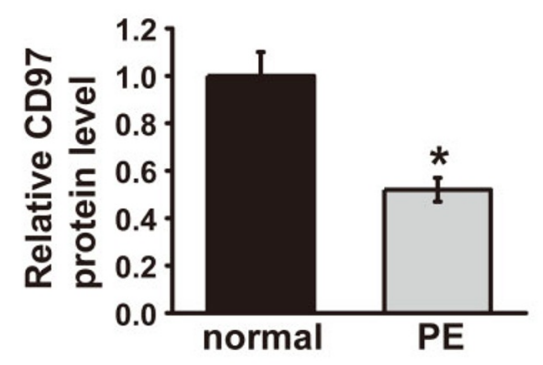

B
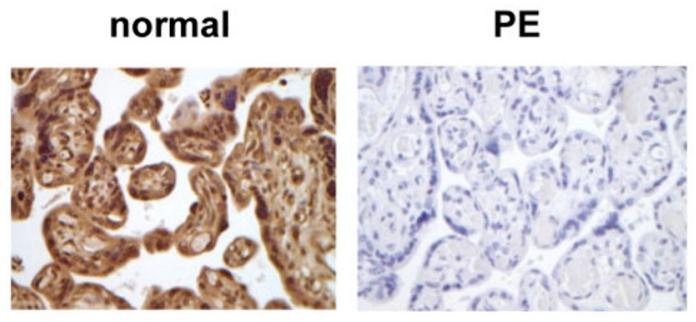

D

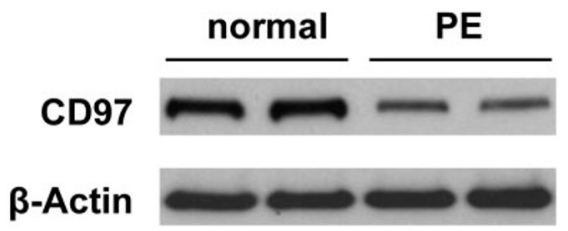

C

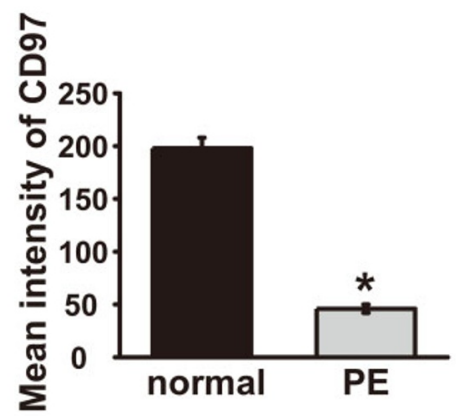

E

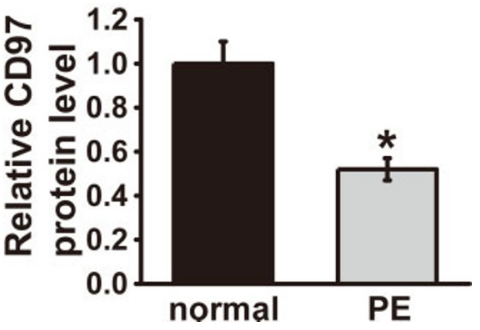

Figure I. The CD97 was decreased in placental tissue samples from pregnant women with PE. A, Quantitative real-time PCR analysis shows significantly decreased CD97 messenger RNA expression in the PE group compared to the healthy control group. B, Immunostaining of CD97 in placentas from the PE and healthy normal groups. C, Mean immunostaining density of CD97 in placentas from the PE and healthy normal groups. Normal: 200 (0.06), PE: 49 (0.04). D, Representative Western blot of CD97 and $\beta$-actin protein levels. E, Relative CD97 protein expression as assessed by densitometry analysis and normalized to $\beta$-actin protein expression. Bars indicate the mean (SD) of 3 independent experiments. $* P<$ .05 compared to normal group. PE indicates preeclampsia; qRT-PCR, quantitative real-time PCR.

regulatory role of CD97 on FOXC2 expression in HTR-8/ SVneo cells. Western blot results showed that the expression level of FOXC2 in trophoblast cells transfected with CD97 siRNAs (si-CD97) was significantly reduced than nontargeting siRNA control (Figure 5A and B), supporting the possible correlation between $\mathrm{CD} 97$ and $\mathrm{FOXC2}$.

\section{FOXC2 Inhibitor Suppresses PI3KIAkt/mTOR Signaling Pathway}

As described above, CD97 showed regulation on both PI3K/ Akt/mTOR signaling pathway and FOXC2 protein expression, and now we were interested to examine how these 2 signals were related. A specific FOXC2 inhibitor was incubated with HTR-8/SVneo cells for 48 hours, and we found the protein levels of PI3K, p-Akt, and p-mTOR were downregulated significantly by FOXC2 inhibitor when compared to control inhibitor (Figure 6A and $\mathrm{B}$ ).

\section{Mammalian Target of Rapamycin Inhibitor Inhibited the Cell Invasion of HTR-8/SVNeo Cells}

To verify the relationship between PI3K/AKT/mTOR pathway and cell invasiveness, we used nVP-BeZ235, a PI3K/mTOR Inhibitor, to treat HTR-8/SVNeo cells. It was found that protein expressions of $\mathrm{p}-\mathrm{AKT}$ and $\mathrm{p}-\mathrm{mTOR}$ were significantly downregulated after nVP-BeZ235 treatment (Figure 7A and B). Our results further showed that the application of nVP-BeZ235 significantly inhibited the cell invasion of CD97-expressiong cells (Figure 7C and D), suggesting the important roles of $\mathrm{PI} 3 \mathrm{~K} / \mathrm{AKT} / \mathrm{mTOR}$ pathway.

\section{FOXC2 Inhibition Downregulated the Cell Invasion of HTR-8/SVNeo Cells}

Likewise, to further verify the relationship among CD97, FOXC2, and PI3K/AKT/mTOR pathway, we used Si-FOXC2 


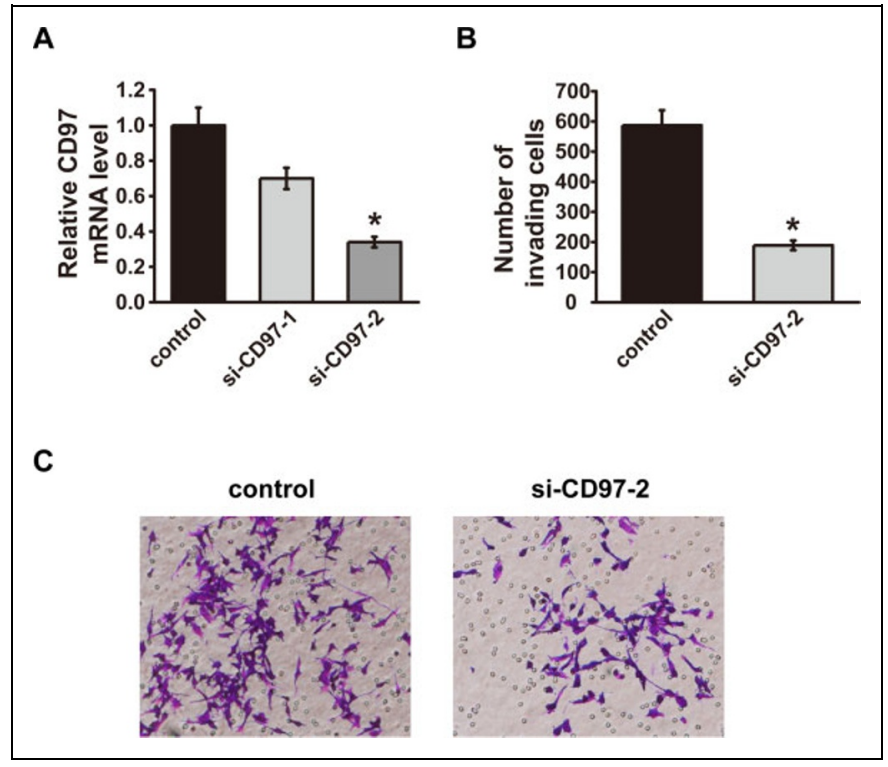

Figure 2. The Si-CD97 inhibits HTR-8/SVneo cell invasion. A, Quantitative real-time PCR analysis of CD97 messenger RNA expression in HTR-8/SVneo cells after transfection with CD97 siRNAs or nontargeting siRNA for 24 and 48 hours. B. The quantitative analysis of invasive HTR-8/SVneo cells in both groups. C, Representative images of Matrigel invasion assays in HTR-8/SVneo cells. Bars indicate the mean (SD) of 3 independent experiments. $* P<.05$ compared to the control group. $\mathrm{qRT}-\mathrm{PCR}$ indicates quantitative real time-PCR.

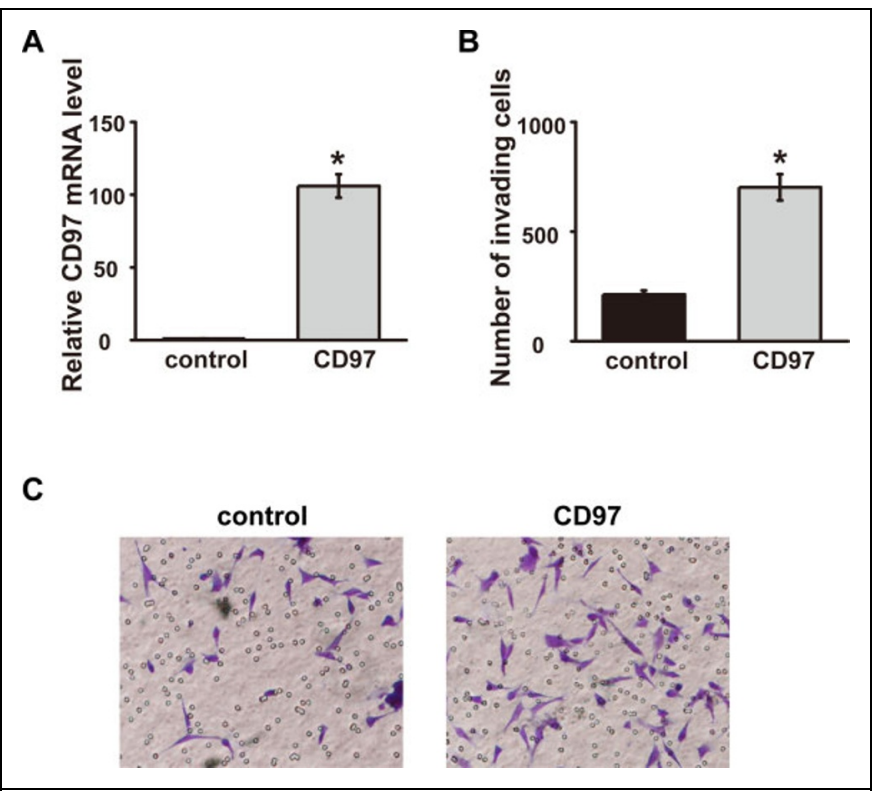

Figure 3. The CD97 overexpression promotes HTR-8/SVneo cells invasion. A, Quantitative real-time PCR analysis of CD97 messenger RNA expression in HTR-8/SVneo cells after transfection of CD97 plasmid or empty vector control for 48 hours. B, The quantitative analysis of invasive HTR-8/SVneo cells in both groups. C, Representative images of Matrigel invasion assays in HTR-8/SVneo cells. Bars indicate the mean (SD) of 3 independent experiments. $* P<.05$ compared to the control group. $\mathrm{QRT}$-PCR indicates quantitative real time-PCR.

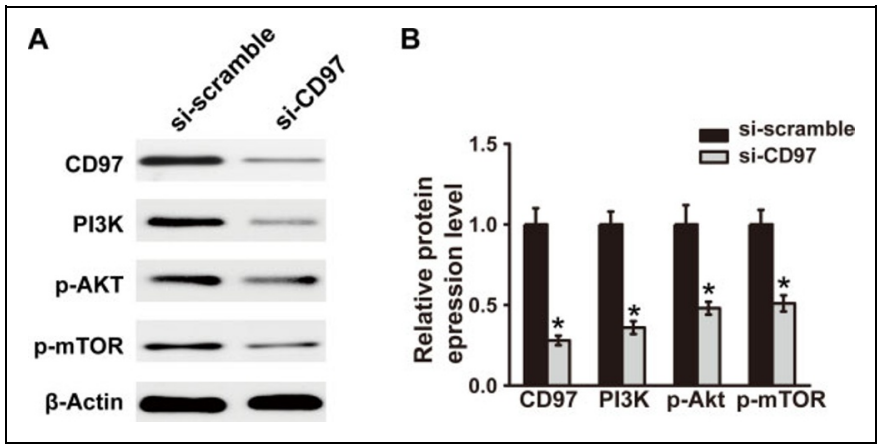

Figure 4. The CD97 regulates the $\mathrm{PI} 3 \mathrm{~K} / \mathrm{Akt} / \mathrm{mTOR}$ signaling pathway. A, Western blot analysis of PI3K/Akt/mTOR components of HTR-8/SVneo cells transfected with CD97 siRNAs (si-CD97) and nontargeting siRNA. B, Quantification of Western blot was performed using Imagej. Bars indicate the mean (SD) of 3 independent experiments. $* P<.05$ compared to the control group. mTOR indicates mammalian target of rapamycin; PI3K, phosphatidylinositol-3-kinase.

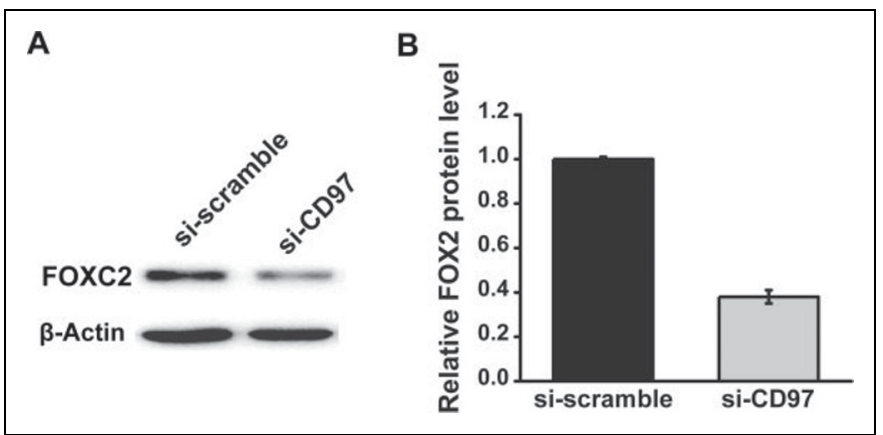

Figure 5. The CD97 regulates the expression of FOXC2. A, FOXC2 expression in HTR-8/SVneo cells transfected with CD97 siRNAs (siCD97) and nontargeting siRNA control (si-scramble). B, Quantification of Western blot was performed using Imagej. Bars indicate the mean (SD) of 3 independent experiments. $* P<.05$ compared to the siscramble group.

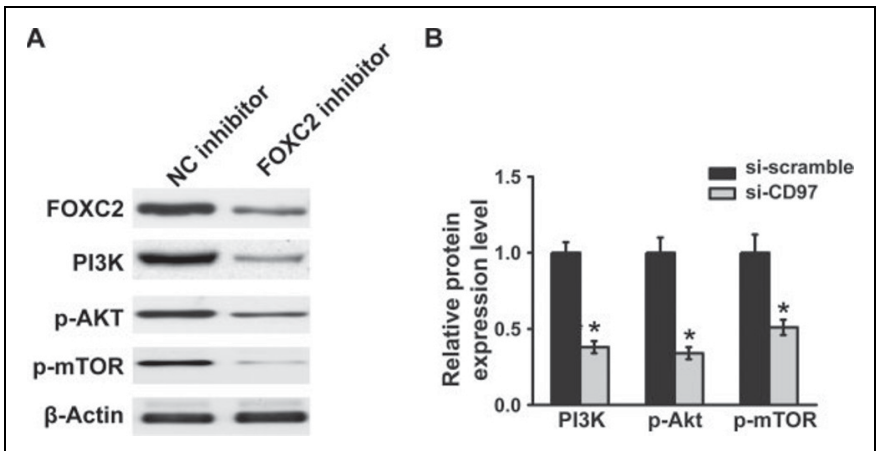

Figure 6. The CD97 regulates $\mathrm{PI} 3 \mathrm{~K} / \mathrm{Akt} / \mathrm{mTOR}$ signaling pathway via FOXC2. A, Western blot analysis of FOXC2 and PI3K/Akt/mTOR components in cells treated with FOXC2 inhibitor or negative control. B, Quantification of Western blot was performed using ImageJ. Bars indicate the mean (SD) of 3 independent experiments. $* P<.05$ compared to the NC inhibitor group. mTOR indicates mammalian target of rapamycin; PI3K, phosphatidylinositol-3-kinase. 


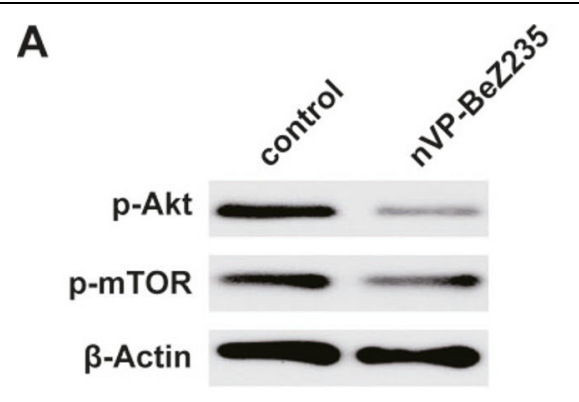

C

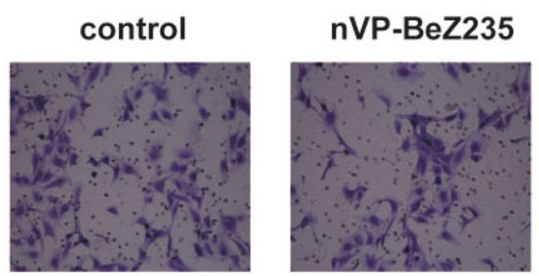

B

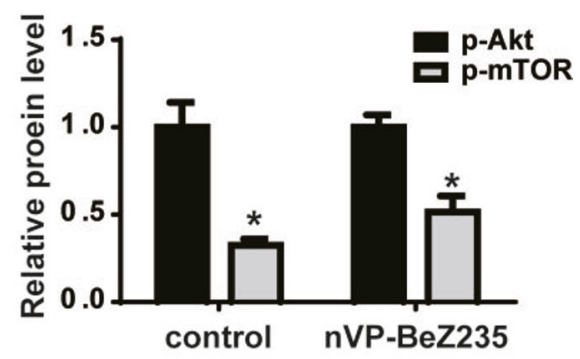

D

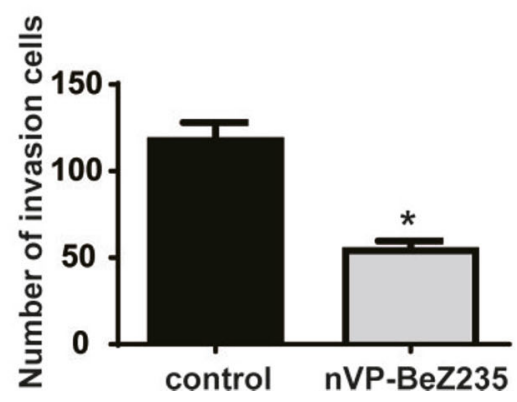

Figure 7. The nVP-BeZ235 treatment inhibits HTR-8/SVneo cells invasion. A, Western blot of PI3K/Akt/mTOR components of HTR-8/SVneo cells treated with nVP-BeZ235. B, Quantification of Western blot. C, Representative images of Matrigel invasion assays in HTR-8/SVneo cells. D, Quantification of invasion number. Bars indicate the mean (SD) of 3 independent experiments. *P $<.05$ compared to the control group. mTOR indicates mammalian target of rapamycin; PI3K, phosphatidylinositol-3-kinase.

to interfere the expression of FOXC2 in HTR-8/SVNeo cells. Our results showed that Si-FOXC2 lowered the expression of FOXC2 significantly (Figure 8A and B). Further, cell invasion assay showed that the addition of Si-FOXC2 significantly inhibited the cell invasion of HTR-8/SVNeo cells (Figure 8C and D), suggesting that FOXC2 serves as target of CD97 on the further regulation of the PI3K/Akt/mTOR signaling pathway and ultimately on the suppression on the trophoblast invasion. Given the results of this study, we speculate that decreasing CD97 expression in placenta could downregulate PI3K/Akt/ mTOR signaling pathway via FOXC2, leading to decreased trophoblast invasion, which contributes to the development of $\mathrm{PE}$ in late pregnancy (Figure 9).

\section{Discussion}

Preeclampsia is a pregnancy-specific disease. Although the etiology of PE remains elusive, the delivery of the placenta has been considered as effective treatment for $\mathrm{PE}^{16}$ Trophoblasts are very important components in the development of placenta, and trophoblast invasion is a strictly regulated physiological event during pregnancy. Defective trophoblast invasion may contribute to many pregnancy-related diseases including $\mathrm{PE}^{19}$ One of the characterizations of normal placental development is the penetration of extravillous trophoblast cells into the maternal decidua and myometrium ${ }^{20}$ The aberrant alteration in the trophoblast invasion ability could severely impact placental function, threatening maternal and fetus health ${ }^{16}$ For instance, it has been reported that late sporadic miscarriage as well as PE may be associated with reduced trophoblast invasion and inadequate transformation of myometrial spiral arteries ${ }^{21}$

The CD97 is a secretive receptor that belongs to the superfamily of 7-span transmembrane molecules ${ }^{22}$ Earlier study showed that expression CD97 expression correlates with the differentiation, migration, and invasion in colorectal tumor cell lines, and tumor cells at the invasion front of carcinomas showed stronger CD-97 staining, indicating the participation of CD97 in tumor migration and invasion ${ }^{13}$ Notably, in the current study, we found expression of CD97 at the mRNA and protein levels in the placentas of PE patients was significantly downregulated when compared to placentas of healthy control group.

Invasiveness is a common characteristic between trophoblasts and carcinomas, although the former is stringently controlled and the latter is not controllable. Based on the data, we speculated that CD97 participates in PE development by promoting trophoblast invasion. To investigate this possibility, Transwell invasion assays were performed along with the knockdown and overexpression of CD97 in cultured human trophoblast cell line HTR-8/SVneo. Our results demonstrated that the invasion of trophoblast cells was strongly inhibited by siRNA-mediated CD97 silencing. Accordingly, overexpression of CD97 promoted human trophoblast invasion, indicating the modulating role of CD97 ontrophoblast function. 


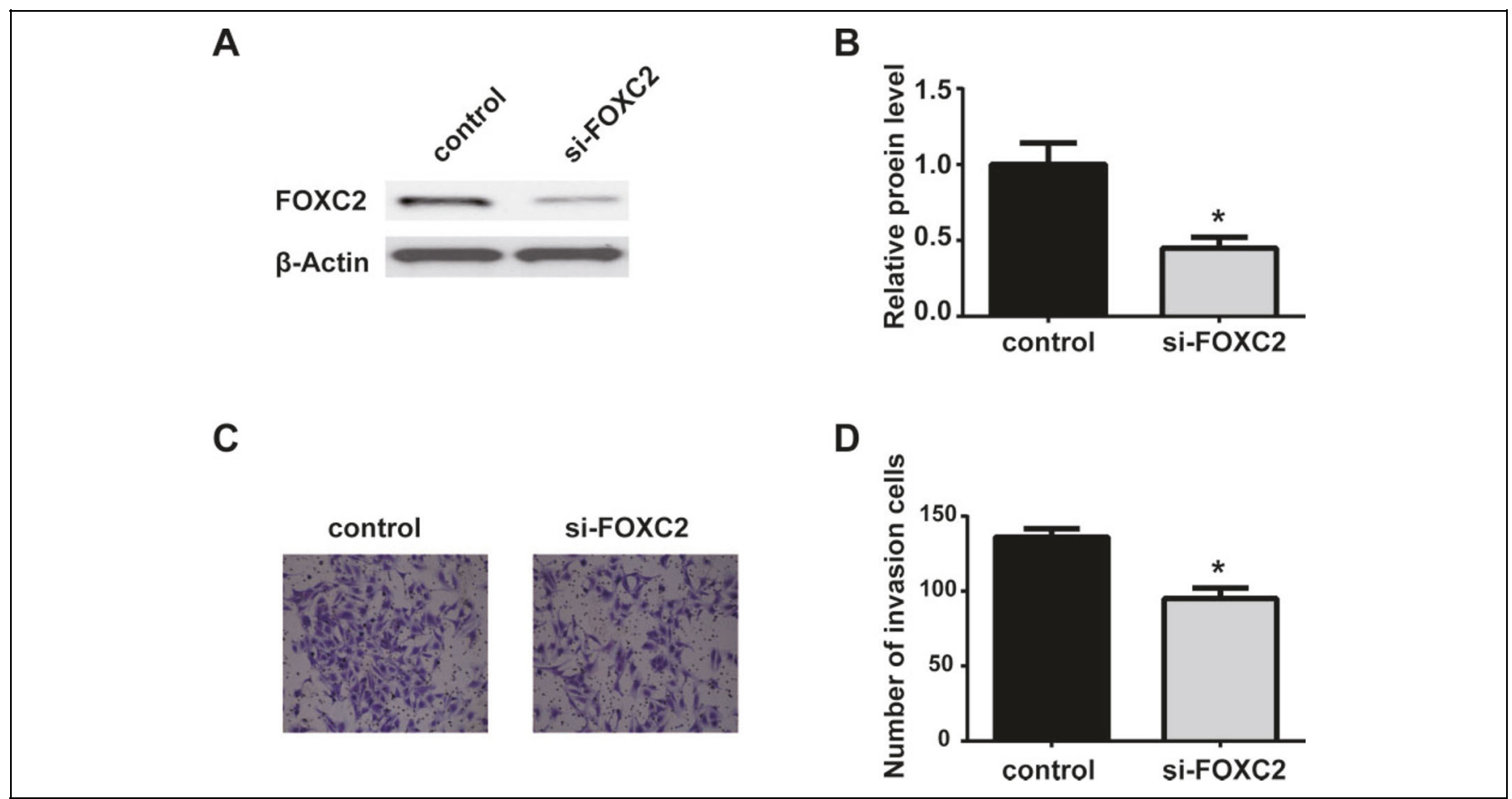

Figure 8. The Si-FOXC2 inhibits HTR-8/SVneo cell invasion. A, Western blot of FOXC2 in HTR-8/SVneo cells transfected with si-control or siFOXC2. B, Quantification of Western blot. C, Representative images of Matrigel invasion assays in HTR-8/SVneo cells. D, Quantification of invasion number. Bars indicate the mean (SD) of 3 independent experiments. $* P<.05$ compared to the control group.

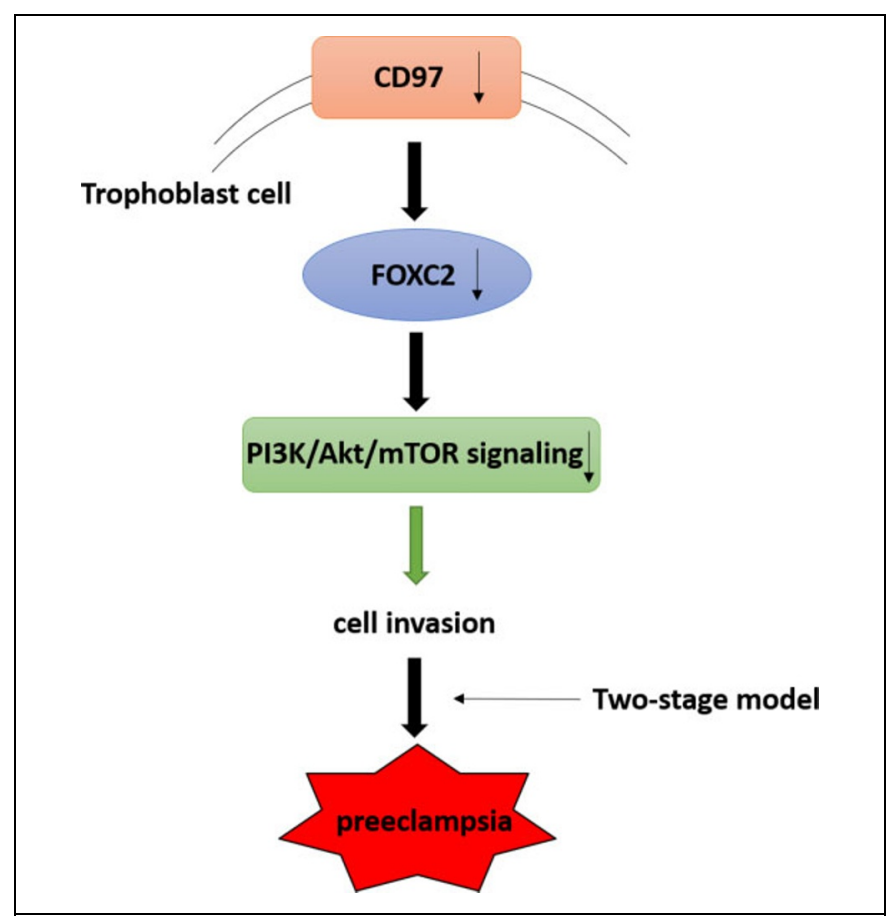

Figure 9. Schematic of the hypothesized molecular mechanism for CD97 contributions to preeclampsia. Given the results of this study, we speculate that decreasing CD97 expression in placenta could downregulate $\mathrm{PI3K} / \mathrm{Akt} / \mathrm{mTOR}$ signaling pathway via FOXC2, leading to decreased trophoblast invasion, which contributes to the development of preeclampsia in late pregnancy. mTOR indicates mammalian target of rapamycin; PI3K, phosphatidylinositol-3-kinase.
Table I. Clinical Characteristics of the Study Sample.

\begin{tabular}{lcc}
\hline & Normal $(\mathrm{n}=2 \mathrm{I})$ & $\mathrm{PE}(\mathrm{n}=38)$ \\
\hline Maternal age (years) & $30.9(3.9)$ & $3 \mathrm{I} . \mathrm{I}(3.2)$ \\
Gestation age at delivery (weeks) & $38.2(\mathrm{I} .2)$ & $36.8(\mathrm{I} .2)$ \\
Antepartum body mass index $\left(\mathrm{kg} / \mathrm{m}^{2}\right)$ & $25.2(2.3)$ & $26.1(5.5)$ \\
Systolic blood pressure $(\mathrm{mm} \mathrm{Hg})$ & $109.6(8.2)$ & $152.8(3.9)$ \\
Diastolic blood pressure $(\mathrm{mm} \mathrm{Hg})$ & $67.3(6.5)$ & $95.4(8.4)$ \\
Birth weight (g) & $3278.6(472.9)$ & $2117.3(365.8)$ \\
\hline
\end{tabular}

Abbreviation: PE, preeclampsia.

Given that overexpressed CD97 plays important roles in PE progression, the mechanisms underlying these functions required further investigation. Several signaling pathways play key roles in cellular proliferation and invasion. Among them, the PI3K/Akt and the mTOR signaling pathways are both crucial to many aspects of cell growth and survival under both physiological and pathological circumstances. These pathways are activated in PE placentas to regulate the expression of soluble fms-like tyrosine kinase $1^{23}$ In addition, the desensitization of PI3K/Akt pathway that underlies endothelial dysfunction was found in PE, promoting the expression of soluble endoglin, an antagonist of transforming growth factor $\beta$ signaling $^{24}$ The PI3K/Akt and the mTOR signaling pathways are generally so closely interconnected that they could be regarded as a unique pathway ${ }^{25}$ In the current study, we found the lower level of CD97 mediated by si-CD97 in HTR-8/SVneo cells was 
related to the significantly downregulated protein levels of PI3K, p-Akt, and p-mTOR. We speculated that the regulation of CD97 on the PI3K/Akt/ mTOR pathway served to reveal the potential mechanism of action of CD97 on trophoblast invasion.

The FOXC2 is an important transcription factor of Forkhead box family, which is also involved in the tumor cells invasion ${ }^{26}$ It has been reported that FOXC2 overexpression remarkably strengthened the adhesion and invasion abilities of normal trophoblast cells ${ }^{18} \mathrm{We}$ further explore the regulatory role of CD97 on FOXC2 expression in HTR-8/SVneo cells. Western blot results showed that the expression level of FOXC2 in trophoblast cells transfected with CD97 siRNAs (si-CD97) was significantly reduced than nontargeting siRNA control, supporting the possible correlation between CD97 and FOXC2. It has been recently revealed that $\mathrm{FOXC} 2$ enhances preadipocytes proliferation and inhibits preadipocytes apoptosis by activating the $\mathrm{Akt} / \mathrm{mTORC} 1$ and ERK/mTORC1 signaling pathways ${ }^{27}$ Herein, we consistently demonstrated that CD97 targeted FOXC2 via PI3K/Akt/ mTOR signaling pathway in trophoblasts. Further, a specific FOXC2 inhibitor was shown to interfere PI3K/Akt/mTOR signaling pathway in cultured trophoblast cells, supported by the downregulation of the expression of all of $3 \mathrm{PI} 3 \mathrm{~K}$, Akt, and mTOR proteins.

One limitation in our study was that only one trophoblast cell line HTR-8/SVneo was utilized for the valuation of CD97 in trophoblast invasion. It has been shown that selection of cell type can have significant impacts on different research purposes. For instance, among the tested cell lines, the choriocarcinoma cell line BeWo is best option for studying syncytial fusion ${ }^{28}$ Since previous research showed that HTR-8/SVneo cell line contains 2 heterogeneous cell populations including trophoblast and stromal/mesenchymal cells, ${ }^{29}$ it would be worthwhile to validate the observations in the current study using different human trophoblast cell lines in the future directions.

\section{Conclusions}

In conclusion, CD97 mRNA and protein expression were downregulated in preeclamptic placentas. Furthermore, our results indicated that $\mathrm{CD} 97$ promoted trophoblast invasion by targeting FOXC2 via PI3K/Akt/mTOR signaling pathway. The present study revealed a potential function of CD97 as well as underline mechanism in the pathogenesis of $\mathrm{PE}$, indicating that CD97 is a potential target for PE intervention.

\section{Authors' Note}

Huaxiang Shen and Minfei Jin contributed equally.

\section{Declaration of Conflicting Interests}

The author(s) declared no potential conflicts of interest with respect to the research, authorship, and/or publication of this article.

\section{Funding}

The author(s) disclosed receipt of the following financial support for the research, authorship, and/or publication of this article: This work was supported by National Natural Science Foundation of China (No. 81873816); Natural science foundation of Shanghai Municipal Health and Family planning Commission (No. 201640366).

\section{References}

1. Abalos E, Cuesta C, Grosso AL, Chou D, Say L. Global and regional estimates of preeclampsia and eclampsia: a systematic review. Eur J Obstet Gynecol Reprod Biol. 2013;170(1):1-7.

2. Roberts JM, Hubel CA. The two stage model of preeclampsia: variations on the theme. Placenta. 2009;30(suppl A):S32-S37.

3. Rajakumar A, Whitelock KA, Weissfeld LA, Daftary AR, Markovic N, Conrad KP. Selective overexpression of the hypoxiainducible transcription factor, HIF-2alpha, in placentas from women with preeclampsia. Biol Reprod. 2001;64(2):499-506.

4. Laresgoiti-Servitje E, Gomez-Lopez N, Olson DM. An immunological insight into the origins of pre-eclampsia. Hum Reprod Update. 2010;16(5):510-524.

5. Hiby SE, Walker JJ, O'Shaughnessy KM, et al. Combinations of maternal KIR and fetal HLA-C genes influence the risk of preeclampsia and reproductive success. J Exp Med. 2004;200(8): 957-965.

6. Hansson SR, Naav A, Erlandsson L. Oxidative stress in preeclampsia and the role of free fetal hemoglobin. Front Physiol. 2014;5:516.

7. Meekins JW, Pijnenborg R, Hanssens M, McFadyen IR, van Asshe A. A study of placental bed spiral arteries and trophoblast invasion in normal and severe pre-eclamptic pregnancies. $\mathrm{Br} J$ Obstet Gynaecol. 1994;101(8):669-674.

8. Naljayan MV, Karumanchi SA. New developments in the pathogenesis of preeclampsia. Adv Chronic Kidney Dis. 2013;20: 265-270.

9. Red-Horse K, Zhou Y, Genbacev O, et al. Trophoblast differentiation during embryo implantation and formation of the maternal-fetal interface. J Clin Invest. 2004;114(6):744-754.

10. Steegers EA, von Dadelszen P, Duvekot JJ, Pijnenborg R. Preeclampsia. Lancet. 2010;376(9741):631-644.

11. Li C, Liu DR, Li GG, et al. CD97 promotes gastric cancer cell proliferation and invasion through exosome-mediated MAPK signaling pathway. World J Gastroenterol. 2015;21(20): 6215-6228.

12. Mallawaaratchy DM, Buckland ME, McDonald KL, et al. Membrane proteome analysis of glioblastoma cell invasion. $J$ Neuropathol Exp Neurol. 2015;74(5):425-441.

13. Steinert M, Wobus M, Boltze C, et al. Expression and regulation of CD97 in colorectal carcinoma cell lines and tumor tissues. $\mathrm{Am}$ J Pathol. 2002;161(5):1657-1667.

14. Liu D, Trojanowicz B, Ye L, et al. The invasion and metastasis promotion role of CD97 small isoform in gastric carcinoma. PLoS One. 2012;7(6):e39989.

15. Wang H, Cheng H, Shao Q, et al. Leptin-promoted human extravillous trophoblast invasion is MMP14 dependent and requires the cross talk between Notch1 and PI3K/Akt signaling. Biol Reprod. 2014;90(4):78.

16. Jia RZ, Ding GC, Gu CM, et al. CDX2 enhances HTR-8/ SVneo trophoblast cell invasion by altering the expression of 
matrix metalloproteinases. Cell Physiol Biochem. 2014;34(3): 628-636.

17. Zhu R, Huang YH, Tao Y, et al. Hyaluronan up-regulates growth and invasion of trophoblasts in an autocrine manner via PI3K/ AKT and MAPK/ERK1/2 pathways in early human pregnancy. Placenta. 2013;34(9):784-791.

18. Zhang Y, Zhang Y. Forkhead box C2 promotes the invasion ability of human trophoblast cells through Hedgehog (Hh) signaling pathway. Cell Biol Int. 2018;42(7):859-866.

19. Norwitz ER. Defective implantation and placentation: laying the blueprint for pregnancy complications. Reprod Biomed Online. 2006;13(4):591-599.

20. Chen JZ, Sheehan PM, Brennecke SP, Keogh RJ. Vessel remodelling, pregnancy hormones and extravillous trophoblast function. Mol Cell Endocrinol. 2012;349(2):138-144.

21. Ball E, Bulmer JN, Ayis S, Lyall F, Robson SC. Late sporadic miscarriage is associated with abnormalities in spiral artery transformation and trophoblast invasion. J Pathol. 2006;208(4): 535-542.

22. Gray JX, Haino M, Roth MJ, et al. CD97 is a processed, seventransmembrane, heterodimeric receptor associated with inflammation. J Immunol. 1996;157(12):5438-5447.
23. Park JK, Jeong JW, Kang MY, et al. Inhibition of the PI3K-Akt pathway suppresses sFlt1 expression in human placental hypoxia models in vitro. Placenta. 2010;31(7):621-629.

24. Cudmore MJ, Ahmad S, Sissaoui S, et al. Loss of Akt activity increases circulating soluble endoglin release in preeclampsia: identification of inter-dependency between Akt-1 and heme oxygenase-1. Eur Heart J. 2012;33(9):1150-1158.

25. Porta C, Paglino C, Mosca A. Targeting PI3K/Akt/mTOR signaling in cancer. Front Oncol. 2014;4:64.

26. Kume T, Jiang H, Topczewska JM, Hogan BL. The murine winged helix transcription factors, Foxc1 and Foxc2, are both required for cardiovascular development and somitogenesis. Genes Dev. 2001;15(8):2470-2482.

27. Gan L, Liu Z, Jin W, Zhou Z, Sun C. Foxc2 enhances proliferation and inhibits apoptosis through activating Akt/mTORC1 signaling pathway in mouse preadipocytes. J Lipid Res. 2015;56(8):1471-1480.

28. Rothbauer M, Patel N, Gondola H, Siwetz M, Huppertz B, Ertl P. A comparative study of five physiological key parameters between four different human trophoblast-derived cell lines. Sci Rep. 2017;7(1):5892.

29. Abou-Kheir W, Barrak J, Hadadeh O, Daoud G. HTR-8/SVneo cell line contains a mixed population of cells. Placenta. 2017;50:1-7. 